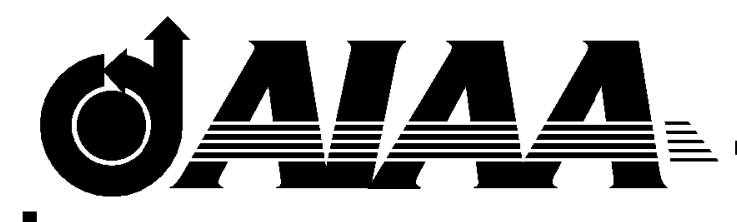

\title{
AIAA 2003-0626 \\ Low Dimensional Tools for \\ Flow-Structure Interaction \\ Problems: Application to Micro Air Vehicles
}

Ryan F. Schmit,

Department of Mechanical and Aeronautical

Engineering

Clarkson University

Potsdam, NY 13699-5725

Mark N. Glauser ,

Department of Mechanical, Aerospace and

Manufacturing Engineering

Syracuse University

Syracuse, NY 13244-1240

Susan A. Gorton

NASA Langley Research Center

Hampton, VA 23666-2199

\section{1st AIAA Aerospace Science Meeting and Exhibit 4 - 9 January, 2003 / Reno, NV}




\title{
Low Dimensional Tools for Flow-Structure Interaction Problems: Application to Micro Air Vehicles
}

\author{
Ryan F. Schmit, \\ Department of Mechanical and Aeronautical \\ Engineering \\ Clarkson University \\ Potsdam, NY 13699-5725 \\ Mark N. Glauser † \\ Department of Mechanical, Aerospace and \\ Manufacturing Engineering \\ Syracuse University \\ Syracuse, NY 13244-1240 \\ Susan A. Gorton \\ NASA Langley Research Center \\ Hampton, VA 23666-2199
}

\begin{abstract}
A low dimensional tool for flow-structure interaction problems based on Proper Orthogonal Decomposition $(P O D)$ and modified Linear Stochastic Estimation $(m L S E)$ has been proposed and was applied to a Micro Air Vehicle (MAV) wing. The method utilizes the dynamic strain measurements from the wing to estimate the $P O D$ expansion coefficients from which an estimation of the velocity in the wake can be obtained.

For this experiment the $M A V$ wing was set at five different angles of attack, from $0^{\circ}$ to $20^{\circ}$. The tunnel velocities varied from 44 to $58 \mathrm{ft} / \mathrm{sec}$ with corresponding Reynolds numbers of 46,000 to 70,000. A stereo Particle Image Velocimetry (PIV) system was used to measure the wake of the $M A V$ wing simultaneously with the signals from the twelve dynamic strain gauges mounted on the wing.

With 20 out of 2400 POD modes, a reasonable estimation of the flow field was observed. By increasing the number of $P O D$ modes, a better estimation of the flow field will occur. Utilizing the simultaneously sampled strain gauges and flow field measurements in conjunction with $m L S E$, an estimation of the flow field with lower energy modes is reasonable. With these results, the methodology for estimating the wake flow field from just dynamic strain gauges is validated.
\end{abstract}

\section{Introduction}

The Micro Air Vehicle (MAV) program was started from a Defense Advanced Research Projects Agency (DARPA) initiative. DARPA wanted an aircraft with a wingspan of no more than six inches to be developed. Government, industry and academic institutions have worked on the problem and each has their own purpose for development. MAVs, in a military role, can be used to show foot soldiers what is over the next hill. For surveillance purposes, the MAV can be used to observe

\footnotetext{
*Sponsor - Graduate Student Research Program

†Associate Fellow, AIAA

Copyright (C) 2003 by the American Institute of Aeronautics and Astronautics. Inc. No copyright is asserted in the United States under Title 17, U.S. Code. The U.S. Government has a royaltyfree license to exercise all rights under the copyright claimed herein for Governmental Purposes. All other rights are reserved by the copyright owner
}

without being seen. For a scientific application, the MAV can be used for the cost-effective remote sensing of atmospheric conditions. ${ }^{1}$

One problem with all MAVs is the low Reynolds number flow that they experience while in flight. Since most MAVs fly in atmospheric conditions, gust and turbulence can affect the flight and controllability of these vehicles. By understanding how the MAVs will react to gust and turbulence, control systems can be developed to reduce the effects of the external loadings. Developing a control system for the motion of the MAV's wing and its wake flow field requires them to be simultaneously sampled because the problems are coupled. With a feedback control system, the enhanced MAV wing might have improved flight characteristics, such as increased maneuverability or stability. In the work presented here the focus is on the sensing aspect 
of the control problem. We use surface strain measurements to estimate the $P O D$ expansion coefficients in the wake, which are then used to estimate the velocity wake flow field when combined with the POD eigenfunctions.

\section{Background}

In 1984, Sneyd $^{2}$ derived the aerodynamic coefficients and stability of a sail with no slack while it was attached to a rigid structure, i.e. fixed leading and trailing edges where camber is accommodated by the stretching of the fabric. He found that sails with no slack have the important property of neutral static stability and if the wings are sufficiently stretchable, they may have a slight degree of positive stability. This property must be important in understanding the flight dynamics of bats and pterodactyls whose wings consist of a tensioned elastic membrane without slack.

In 1995, Smith and Shyy ${ }^{3}$ performed computations of the harmonically forced, unsteady viscous flow over a flexible, two-dimensional membrane wing. The simulation of a harmonically forced free-stream flow also proves to be a useful vehicle for demonstrating some of the more generic features of membrane wing mechanics.

Smith and Wright ${ }^{4}$ used computations of the harmonically forced, unsteady incompressible flow over an extensible flexible membrane airfoil. The role of viscosity was shown to be significant in the harmonically forced unsteady flow about a membrane wing. In the unsteady scenario, the influence on viscosity is enhanced since the acceleration and deceleration of the free stream velocity strongly influences the separation and reattachment of the flow.

In 2001, Dowell and Hall ${ }^{5}$ showed various physical models for a fluid undergoing time-dependent motion, the distinction between linear and nonlinear models, time-linearized models and their solutions in the frequency or time domain. They extended treatment of the modal character of time-dependent flows and the construction of reduced-order models base on an expansion in terms of fluid modes. Other works by Naudascher and Rockwell ${ }^{6}$ and Dowell ${ }^{7}$ on this subject offer better understanding of problems that deal with the flow and structure.

Lumley, ${ }^{89}$ developed the Proper Orthogonal Decomposition (POD) technique as a tool to identify the most energetic coherent structures, or eddies, contained within a turbulent flow field. The first validation of the $P O D$ was performed by two of his graduate students. Payne ${ }^{10}$ found that the energy associated with the "dominant eddy" in the wake of a cylinder was not significantly greater than any of the other structures. Bakewell and Lumley ${ }^{11}$ then applied the $P O D$ to the near wall flow region of a pipe flow and showed that the "dominant eddy" contained roughly $90 \%$ of the energy. These initial efforts suggested the power of the $P O D$ could be a useful tool for the study of turbulent flows.

In 1998 Bonnet et al, ${ }^{12,9}$ provided a comparison of a few tools for the decomposition and analysis of turbulent flows that placed an emphasis on the detection of large scale coherent structures. Some techniques that were covered are Proper Orthogonal Decomposition, Linear Stochastic Estimation (LSE) and Conditional Sampling Approaches.

In 2002, Taylor and Glauser ${ }^{13}$ performed an experiment with a separating shear flow in the ActiveWING facility at Clarkson University. This facility is a twodimensional backward facing ramp with an adjustable flap above the ramp that creates conditions from fully separated to fully attached flow on the ramp. Particle Image Velocimetry (PIV) and wall pressure were simultaneously sampled at a number of Reynold numbers and many angles of attack. Moreover, the instantaneous flow field can be reconstructed for any flow condition measured using the instantaneous pressure measurements on the wall.

\section{MAV Wing}

The dimensions of the MAV wing used for the experiment are shown in Figure 1. The span and chord lengths of the wing are 5.75 and 3 inches, respectively. The aluminum frame consisted of one spar and four ribs. The frame has a $0^{\circ}$ camber. An inch of the frame was used to hold the wing into the mounting block. The mounting block was then attached to the tunnel floor. The wing's angle of attack can be adjusted from $-20^{\circ}$ to $20^{\circ}$ by rotating the mounting block. To reduce the influence of the tunnel boundary layer, the wing was mounted $1 / 2$ inch above the tunnel floor.

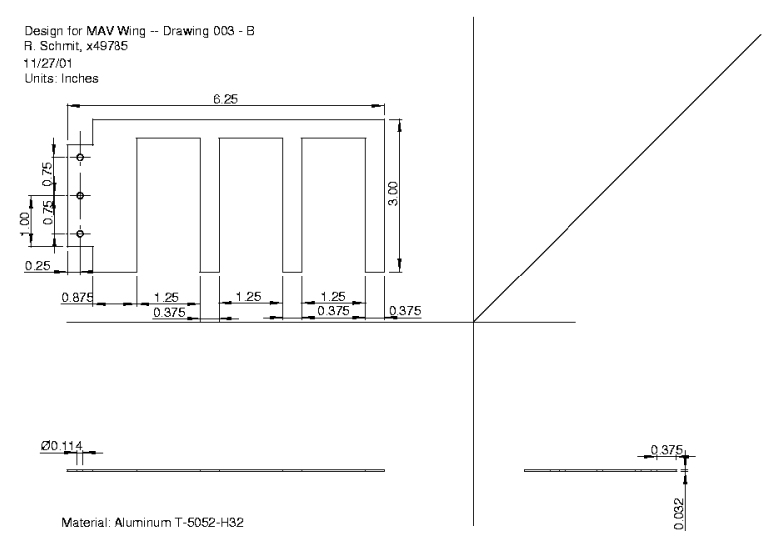

Fig. 1 Dimensions of MAV Wing

A rectangular latex membrane 3 by 5.25 inches, was used for the skin of the wing. Three membrane regions, 2.625 by 1.25 inches, were formed between each pair of ribs. Super $77^{T M}$ a spray adhesive from $3 M^{T M}$ was used to adhere the latex membrane to the frame. 


\section{Experiment}

The experiment was conducted in the NASA Langley 15-Inch Low Speed Wind Tunnel. This tunnel is a low-speed, closed return, atmospheric facility used primarily for fundamental flow physics research. The maximum flow speed in the test section is approximately $115 \mathrm{ft} / \mathrm{sec}$. All data included here was taken in the test section closest to the inlet.

A Laranko Stereo Particle Image Velocimetry, ${ }^{15}$ PIV, was employed to capture the wake flow field of the MAV wing. The system uses a $400 \mathrm{~m} J$ YAG Laser to illuminate the seed particles in the flow field. Two $2 K x 2 K$ digital cameras are used to capture the flow. Mineral oil was atomized and was used for the seed particulate which has the diameter of .1 to $5 \mu \mathrm{m}$ 's and can follow the flow up to the frequency of $1 \mathrm{kHz}$.

Using I-Deas Finite Element Modeling Program the placement of the strain gauges were determined using a Normal Mode Dynamic Solver. A total of six locations of high strain have been determined. At each of these locations a bi-axial strain gauge were placed. Figure 2 shows the placement of all twelve strain gauges, they are numbered for identification purposes.

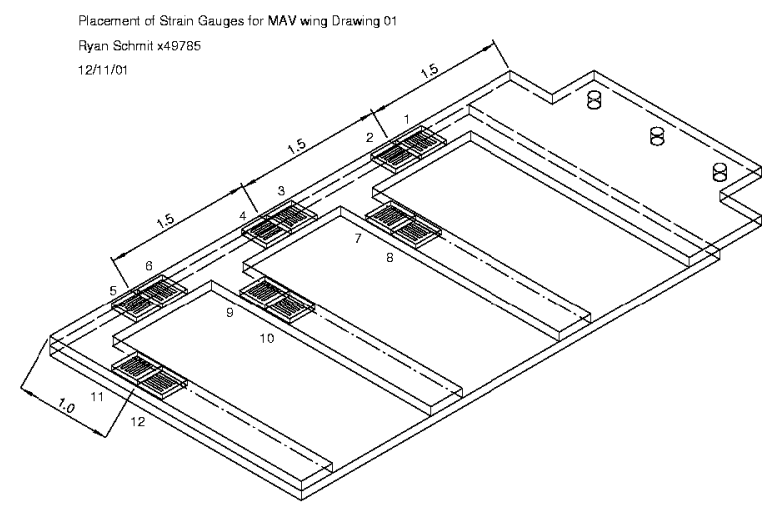

Fig. 2 Placement of Strain Gauges on the Frame

Each strain gauge used has a gauge length and width of $3 \mathrm{~mm}^{\prime} \mathrm{s}$ and of $2 \mathrm{~mm}^{\prime} \mathrm{s}$, respectively. The matrix length and width are $7 \mathrm{~mm}^{\prime} s$ and $4 \mathrm{~mm}^{\prime} s$, respectively. The Gauge Factor of $2.120 \pm 0.5 \%$ at $24^{\circ} \mathrm{C}$. The resistance is $350.0 \pm 0.3 \%$ ohms at $24^{\circ} \mathrm{C}$ with a Transverse sensitivity of $(+0.7 \pm 0.2) \%$ at $24^{\circ} \mathrm{C}$. There are three wire tabs so that temperature compensation can be taken into account. The strain gauges were placed on the aluminum with an adhesive. The wires are then lightly adhered to the wings so that they will not vibrate in the flow.

To determine the instantaneous wake flow field of the MAV wing, the PIV system and strain gauges were used. Because the PIV system is independent of the Zonic data acquisition system, used for the strain gauge measurements, a method to connect the systems had to be developed, enabling the simultaneous sampling of the strain gauges and the wake flow field to occur. The methodology that was developed involved taking the Q-switch signal from the first PIV laser and allowing the DAQ system to sample the signal and can be seen in Figure 3. The Q-switch signal works on a Transistor-Transistor Logic (TTL) pulse of +5 volts $10 \mathrm{~Hz}$ square wave, that is sent from the control box to the lasers. It can also be started with an external trigger that is set with a TTL pulse from a signal generator. Every time a +5 volt pulse is sent from the controller to the lasers, the lasers will fire. A trigger setting of a negative slope and $50 \%$ change in the full scale voltage was used to start the sampling of the strain gauges. Because the Q-switch is being sampled simultaneously with the strain gauges, the PIV images are then considered to be simultaneously sampled with the strain gauges.

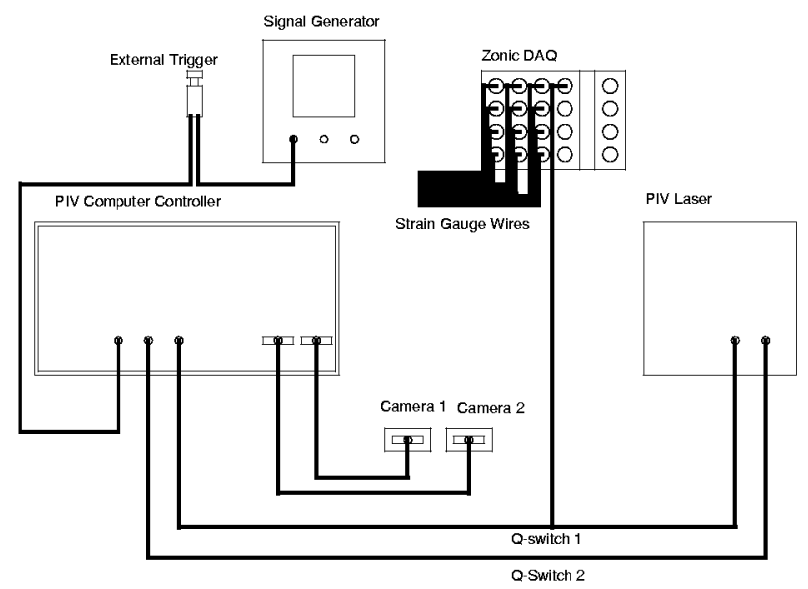

Fig. 3 Wire Diagram of Simultaneous Sampling Setup

For the experiment the MAV wing's angle of attack was changed from $0^{\circ}$ to $20^{\circ}$ at $5^{\circ}$ intervals. The tunnel's air speed was changed from $44 \mathrm{ft} / \mathrm{s}$ to $58 \mathrm{ft} / \mathrm{s}$ which gives a Reynolds number of 40,000 and 70,000 respectively. The PIV images were captured at $c / 2$ and $2 c / 3,1.5$ and 2.0 inches, respectively downstream of the wing. With five different angles of attack, two tunnel airspeeds, and two PIV image planes, a total of 20 runs are required for this experiment.

\section{Analysis}

In 1967 Lumley $^{8}$ proposed the Proper Orthogonal Decomposition, $P O D$, as an unbiased technique for studying coherent structures in turbulent flows. The $P O D$ is a mathematical approach based on the Karhunen-Loeve expansion to decompose the fluctuating velocity field into a finite number of empirical functions.

Adrian $^{16}$ proposed that stochastic estimation could be applied to unconditional data. This method used what Adrian called a "conditional eddy" to form a technique for estimating the flow field given the presence of this defined "conditional eddy." Cole et al. ${ }^{17}$ 
then proposed that the instantaneous velocity field could be used, instead of defining an arbitrary indicator such as Adrian's "conditional eddy."

In 1994, Bonnet et al. ${ }^{18}$ presented the Complementary Technique which combines the $L S E$ and the $P O D$ into a tool for the development of time varying lowdimensional descriptions of the flow.

Taylor and Glauser ${ }^{13}$ proposed the use of Global $P O D$ to reconstruct the instantaneous flow field from the instantaneous wall pressure measurements in a flow over a backward facing ramp with a cyclic flap over the ramp which allows for a dynamically changing adverse pressure gradient. In 2002, Schmit $^{19}$ extended the work of Taylor to reconstruct the instantaneous velocity wake flow field from the instantaneous motion of the $M A V$ wing. For this experiment, twelve judiciously placed strain gauges and a stereo PIV system were used to measure simultaneously the instantaneous strain and three components of velocity in the wake of the $M A V$.

The analysis methodology can be summarized as:

1. From a $2 D-3$ Component velocity measurement, determine the two-point correlation tensor, Equation 1 , at each PIV stream-wise location, $\boldsymbol{x}_{0}$, measured in the wake.

$R_{i j}\left(x_{0}, y, y^{\prime}, z, z^{\prime}\right)=\sum_{k=1}^{M}\left(\frac{\left.\overline{U_{i}\left(x_{0}, y, z\right), U_{j}\left(x_{0}, y^{\prime}, z^{\prime}\right.}\right)}{M}\right)_{k}$

With Equation 1 and following the Hilbert-Schmidt theory $\left(\right.$ Lumley $^{8}$ ), a discrete set of solutions, Equation 2 , can be obtained if random fluctuations occur over a finite domain.

$$
\begin{gathered}
\int_{D} R_{i j}\left(x_{0}, y, y^{\prime}, z, z^{\prime}\right) \phi_{j}^{(n)}\left(x_{0}, y^{\prime}, z^{\prime}\right) d y^{\prime} d z^{\prime}= \\
\lambda^{(n)}\left(x_{0}\right) \phi_{i}^{(n)}\left(x_{0}, y, z\right)
\end{gathered}
$$

Equation 2 is solved numerically for the eigenfunctions of the two-point correlation. The $\phi_{i}^{(n)}$ can then be used to reconstruct the original fluctuation quantities with Equation 3.

$$
U_{i}\left(x_{0}, y, z, t\right)=\sum_{n=1}^{m} a_{n}\left(x_{0}, t\right) \phi_{i}^{(n)}\left(x_{0}, y, z\right)
$$

The $P O D$ expansion coefficients of the flow, $a_{n}\left(x_{0}, t\right)$ can be determined with Equation 4 , where the $\phi_{i}^{(n) *}$ denotes the complex conjugate of $\phi_{i}^{(n)}$.

$$
a_{n}\left(x_{0}, t\right)=\int_{D} U_{i}\left(x_{0}, y, z, t\right) \phi_{i}^{(n) *}\left(x_{0}, y, z\right) d y d z
$$

2. Using the simultaneously sampled strain gauge measurements and the $P O D$ expansion coefficients, the $L S E$ coefficient, $A_{n_{k}}$, can be determined for each streamwise location by:

$$
\left[\begin{array}{cccc}
\overline{\varepsilon_{1}^{2}} & \overline{\varepsilon_{1} \varepsilon_{2}} & \ldots & \overline{\varepsilon_{1} \varepsilon_{q}} \\
\overline{\varepsilon_{2} \varepsilon_{1}} & \overline{\varepsilon_{2}^{2}} & \ldots & \overline{\varepsilon_{2} \varepsilon_{q}} \\
\vdots & \vdots & \ddots & \vdots \\
\overline{\varepsilon_{q} \varepsilon_{1}} & \overline{\varepsilon_{q} \varepsilon_{2}} & \ldots & \overline{\varepsilon_{q}^{2}}
\end{array}\right]\left[\begin{array}{c}
A_{n 1} \\
A_{n 2} \\
\vdots \\
A_{n q}
\end{array}\right]=\left[\begin{array}{c}
\overline{a_{n} \varepsilon_{1}} \\
\overline{a_{n} \varepsilon_{2}} \\
\vdots \\
\overline{a_{n} \varepsilon_{q}}
\end{array}\right]
$$

3. Finally, estimate the $P O D$ expansion coefficients from the temporal stain gauge measurements, shown in Equation 6, and combine them with the eigenfunctions, to produce the instantaneous estimations of flow field as expressed in Equation 7.

$$
\begin{gathered}
a_{n}{ }^{e s t}\left(x_{0}, t\right)=\sum_{k=1}^{q} A_{n_{k}} \varepsilon_{k}(t) \\
U_{i}\left(x_{0}, y, z, t\right)=\sum_{n=1}^{m} a_{n}^{e s t}\left(x_{0}, t\right) \phi_{i}^{(n)}\left(x_{0}, y, z\right)
\end{gathered}
$$

\section{Results}

The stereo PIV system used for this experiment can measure the instantaneous wake flow field in a twodimensional plane with three components of velocity. Figure 4 shows an instantaneous snapshot of the flow at $44 \mathrm{fps}$, downstream at $c / 2$ and an angle of attack of $15^{\circ}$. The reader is looking downstream, in the $x$ direction, from the MAV wing to the $y z$ plane. Figure 4 contains four separate sets of data which can be seen in Table 1.
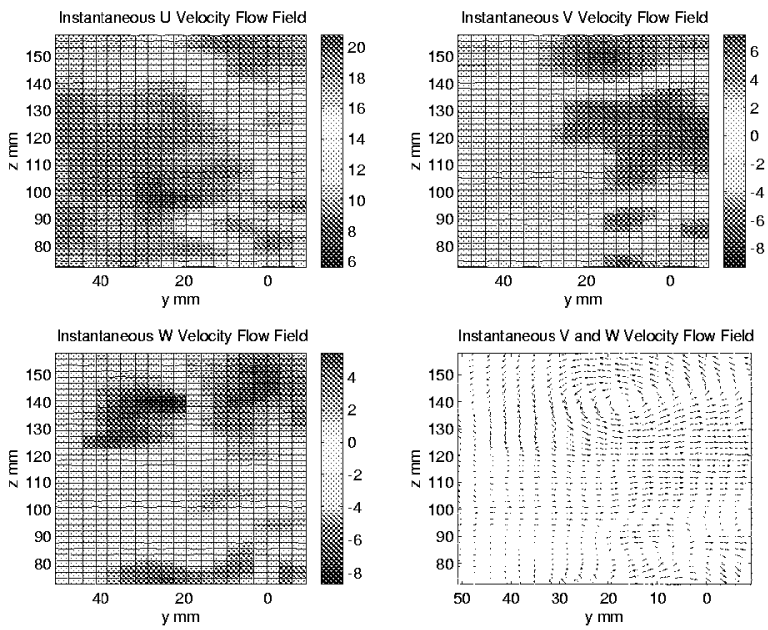

Fig. 4 Instantaneous Snapshot of the Wake Flow Field

\section{Eigenvalues and Eigenvectors}

Once the two-point correlation tensor has been formed the eigenfunction of the tensor can be solved by using Equation 2. One method used to understand the eigenvalues and their importance is to examine the energy contained up to $N P O D$ modes as depicted in 
Upper Left $\quad U$ Component

Upper Right $V$ Component

Lower Left $\quad W$ Component

Lower Right $V$ and $W$ Components

Table 1 Results Setup for Instantaneous Snapshot of the Wake Flow Field

Equation 8. Note that in Equation $8, N$ will eventually go to $m$ the total number of $P O D$ modes.

$$
\sum_{n=1}^{N} \lambda^{(n)}
$$

We solved the eigenvalue problem with and without the mean velocity flow field. Figure 5 shows the summation of energy for each set of conditions with the mean included. Figure 6 shows the summation of energy for each set of conditions without the mean included.

As seen in Figure 5 the mean mode has about 95 to $97 \%$ of the total energy of the flow, this is out of 2400 $P O D$ modes. From Figure 6 the summation of the turbulent modes show that the $1^{\text {st }}$ mode has about $20 \%$ of the total energy, this is out of 2399 POD modes. In both cases, as $N$ increase in Equation 8 the total energy available in that mode is less than the one before. It takes approximately $400 P O D$ modes in both cases to obtain nearly $100 \%$ of the energy available in the flow. However, with only 20 POD modes, we obtain more than $99 \%$ of the total energy when we include the mean as seen in Figure 5 and more than $70 \%$ of the total energy when we examine the fluctuations only as shown in Figure 6. These results give a good indication that this flow has a low-dimensional description.
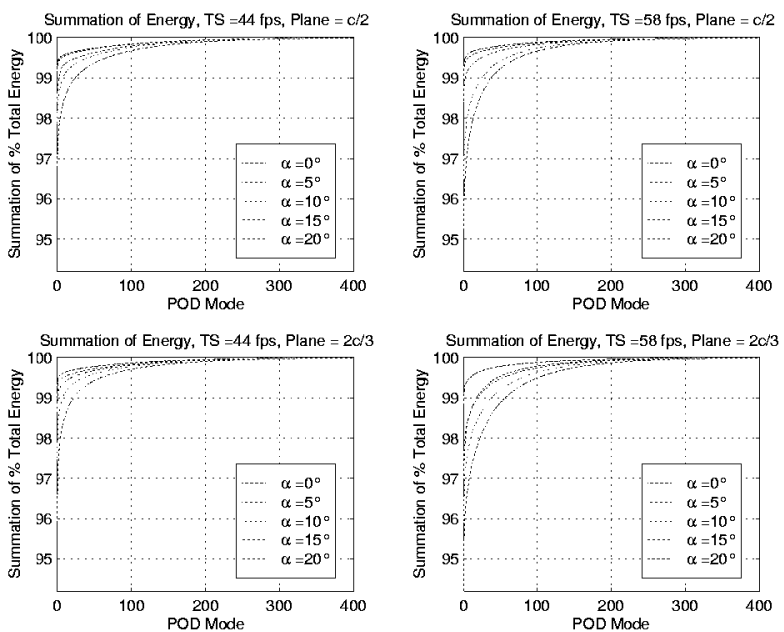

Fig. 5 Summation of Energy per POD mode with mean included.

Figures 7 to 10 show the first $4 P O D$ eigenvectors, $\phi_{i}^{(n)}$, with the mean included. Because the $1^{\text {st }} P O D$ mode contains up to $95 \%$ of the total energy it should
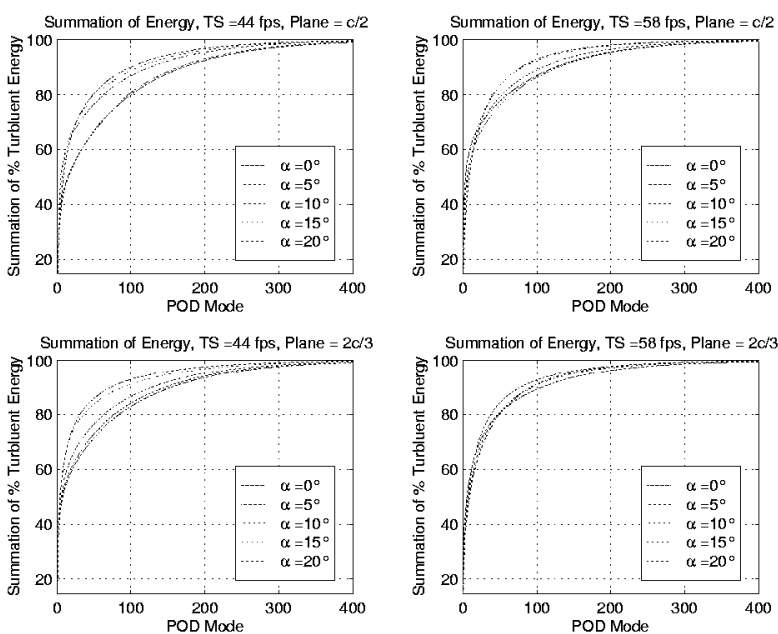

Fig. 6 Sumation of Energy per POD mode without mean included.

come as no surprise that it resembles the mean velocity profile.
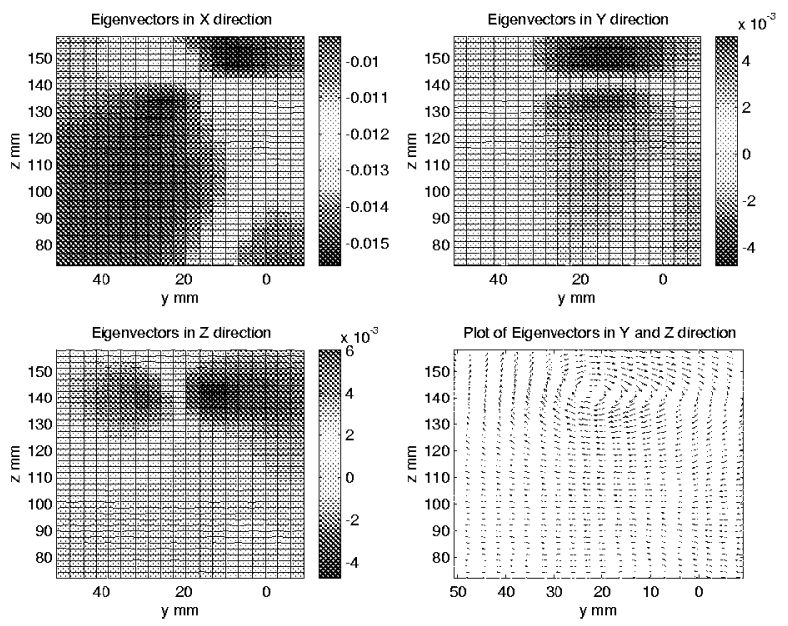

Fig. 7 Eigenvector Mode 1 at $44 f p s, 15^{\circ}$ AOA, $c / 2$

\section{Reconstruction of a Selected Sample}

With the $P O D$ expansion coefficients solved for in Equation 4, the flow field can be reconstructed by utilizing Equation 3. By rebuilding the flow field in this manner a minimum number of $P O D$ modes can be determined for adequately representing the velocity field of that snapshot.

To properly determine the minimum number of $P O D$ modes to be used to rebuild the instantaneous snapshot of the flow field, original snapshots of the wake flow field need to be used for direct comparison. Figure 4 shows instantaneous snapshots of the wake flow field at $15^{\circ}$ angle of attack, with an airspeed of $44 f p s$ at a downstream location of $c / 2$.

By using only 20 out of 2400 POD modes, shown in Figure 11, an excellent reconstruction of the instantaneous snapshot of the wake flow field results. This can 

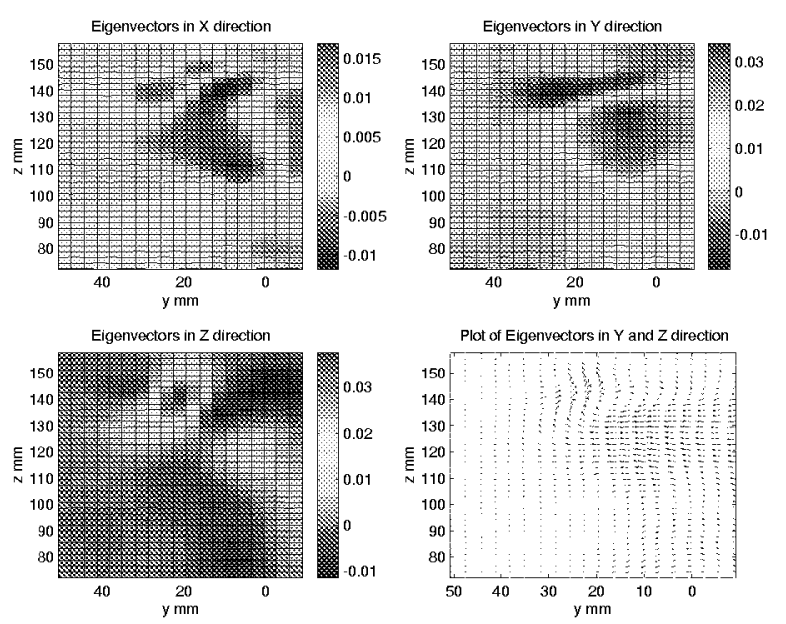

Fig. 8 Eigenvector Mode 2 at $44 \mathrm{fps}, 15^{\circ}$ AOA, $c / 2$
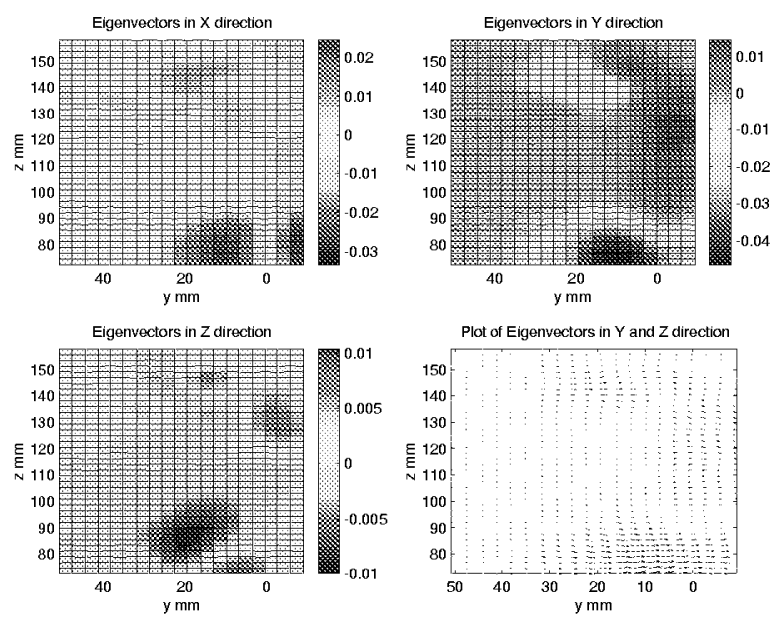

Fig. 9 Eigenvector Mode 3 at $44 f p s, 15^{\circ}$ AOA, $c / 2$
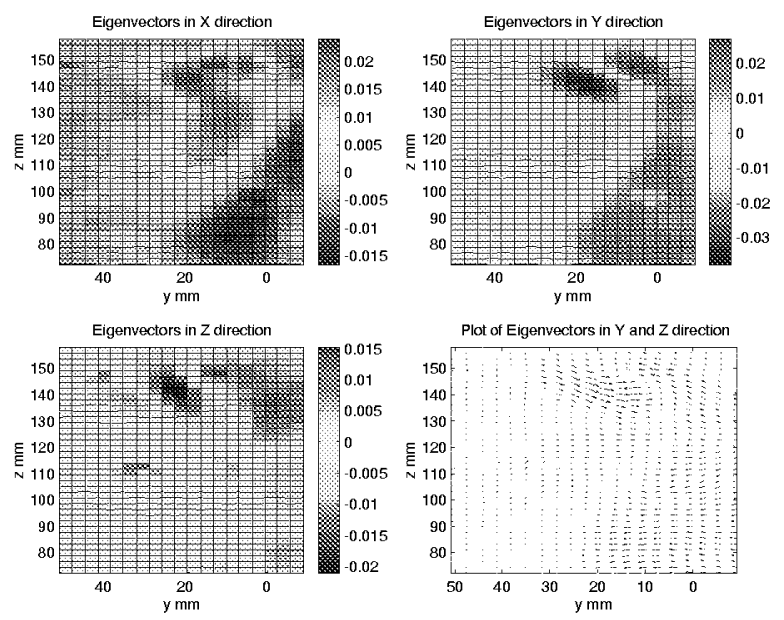

Fig. 10 Eigenvector Mode 4 at $44 \mathrm{fps}, 15^{\circ}$ AOA, $c / 2$

be seen by direct comparison to the results presented in Figure 12 where all 2400 POD modes are included in the reconstruction. Twenty modes were found suf- ficient for all other cases examined. ${ }^{19}$
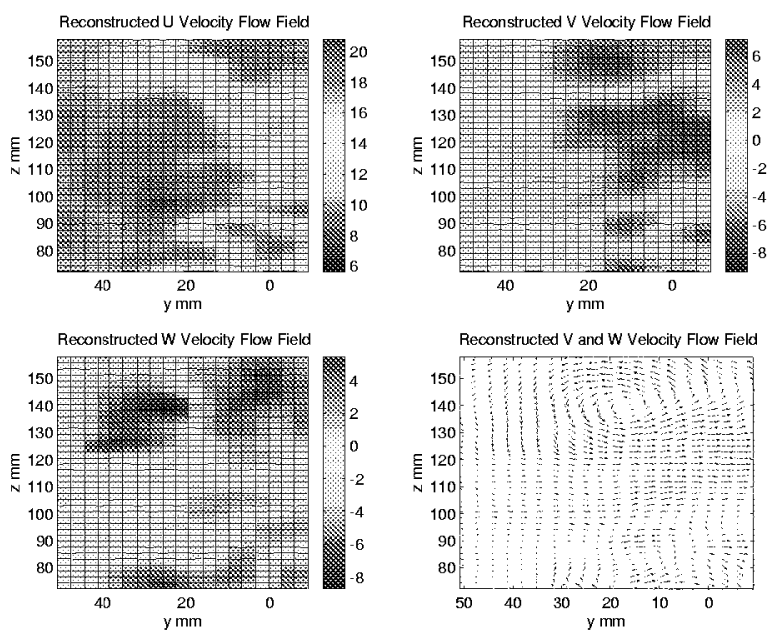

Fig. 11 Rebuild of Instantaneous Snapshot with 20 POD Mode at $\alpha=15^{\circ}$ AOA, airspeed $=44 \mathrm{fps}$, plane $=c / 2$
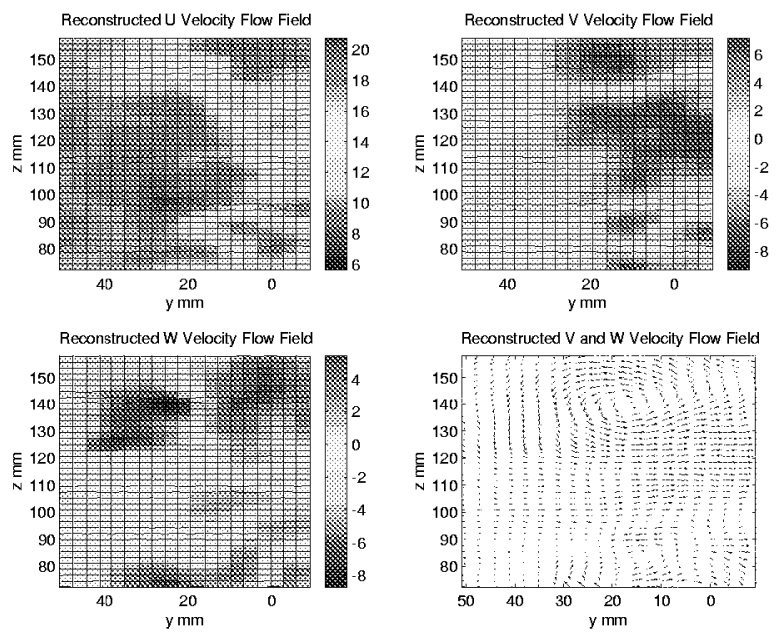

Fig. 12 Reconstruction of the Instantaneous Snapshot with 2400 POD Modes at $\alpha=15^{\circ}$ AOA, airspeed $=44 f p s$, plane $=c / 2$

\section{Results of Estimation Procedure}

If one considers the distance between the trailing edge of the MAV wing to the PIV sampling planes, the simultaneous sampling of the strain gauges and PIV system need to be phased aligned. Because the PIV sampling planes are $c / 2$ and $2 c / 3$ positions downstream from the trailing edge, the flow that is being sampled by the PIV in the wake has already passed over the wing's surface. Or in other words, the eddies that are shed from the MAV wing's surface have to travel a distance downstream before they encounter the PIV sampling plane. To phase align these measurement we define a convective eddy time based on Equation 9 


$$
T_{C E}=x / U_{c}
$$

where $x$ is the distance from the middle of the MAV wing to the plane and $U_{c}$ is the convective velocity that the eddies move at. ${ }^{20}$ The convective eddy time is estimated to be $0.008 \mathrm{sec}$ for the first plane $c / 2$ where $U_{c}=0.7 U_{\infty}$ and $U_{\infty}=44 f p s$.

Figure 13 presents a set of simultaneously sampled strain gauge measurements along with the PIV TTL pulse signal which allows for phase alignment of the strain gauge and PIV data.
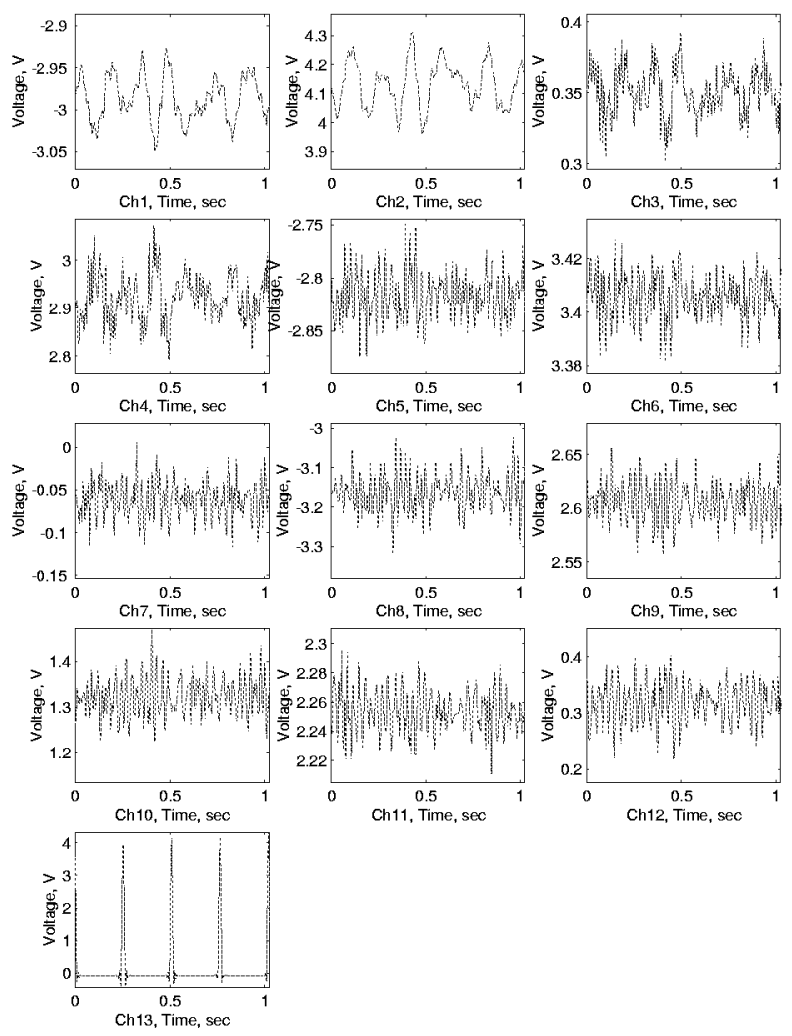

Ch11, Time, sec

Ch12, Time, sec
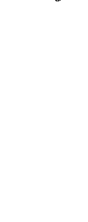

Fig. 13 Simultaneously Sampled Strain Gauge and TTL Pulse Signals.

Figure 14 presents an estimate of the wake flow field using the estimation procedure described by Equations 5, 6 and 7 with 20 POD modes. From the results presented in Figure 14, the estimate of the wake flow field shows that the technique captures much of the low dimensional features of the flow. This can be clearly seen by comparing these results to those presented in Figure 11.

The focus of this work is on the sensing aspect of the control problem. Our proposal for using surface strain measurements to estimate the $P O D$ expansion coefficients in the wake, which are then used to estimate the velocity wake flow field when combined with the $P O D$ eigenfunctions is working to capture the large scale features of the flow.
The $P O D$ itself can be viewed as a filter, the estimation procedure here acts like a filter as well. This can be seen by examining the results shown in Figure 15 where we include in our estimation all 2400 $P O D$ modes. As can be seen from the figure the estimation of the velocity does not improve with the additional $P O D$ Modes. In fact comparing the results from Figure 15 with those of Figure 11 where only 20 $P O D$ modes were used you can directly see the filtering effect of the estimation procedure. The inability of the estimation procedure to capture the finer scales is probably do to the fact the strain gauges do not pick up the signature of the small scales. For control of the large scales in the flow however, this loss of information is not critical.
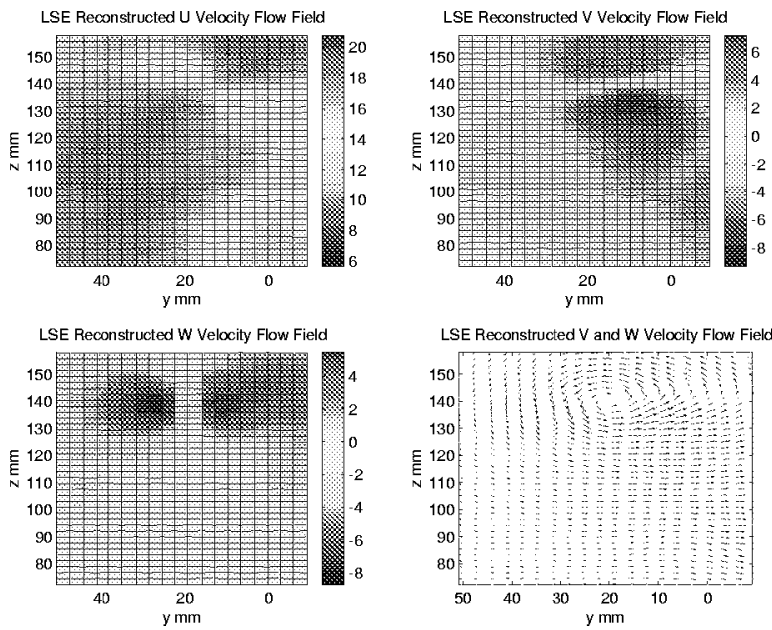

Fig. 14 Estimation of the Wake Flow Field with 20 Modes at $\alpha=15^{\circ}$, airspeed $=44 \mathrm{fps}$, plane $=c / 2$
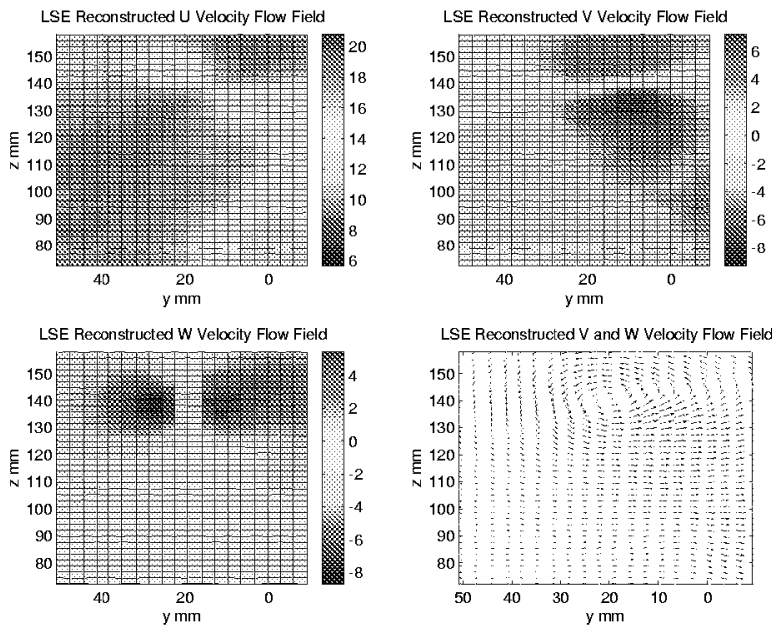

Fig. 15 Estimation of the Wake Flow Field with 2400 Modes at $\alpha=15^{\circ}$, airspeed $=44 \mathrm{fps}$, plane $=c / 2$ 


\section{Conclusions}

A low dimensional tool for flow-structure interaction problems based on Proper Orthogonal Decomposition $(P O D)$ and modified Linear Stochastic Estimation $(m L S E)$ was proposed and applied to a Micro Air Vehicle $(M A V)$ wing. The method utilized the dynamic strain measurements from the wing to estimate the $P O D$ expansion coefficients from which an estimation of the velocity in the wake can be obtained.

With the statistical data base that was required for the low dimensional tool, the statistical quantities for the mean flow field show that the wing tip vortex dominates the flow. From the $P O D$ eigenfunctions, the $1^{\text {st }}$ mode contains $95 \%$ or more of the energy in the wake flow field. This captures nicely the wing tip vortex. With 20 out of 2400 POD modes, a reasonable reconstruction of the instantaneous snapshot of the wake flow field can be obtained.

Utilizing the simultaneously sampled strain gauges and the wake flow field measurements in conjunction with $m L S E$, an estimation of the wake flow field with the lower $P O D$ modes, provides a reasonable estimation of the wake flow field.

From the results shown, using a low dimensional tool for flow-structure interaction provides a valid methodology for estimating the wake flow field from the dynamic strain gauges alone. This provides a starting point for developing control strategies for MAV's where the vehicle itself can morph and essentially become a part of the control system.

\section{References}

${ }^{1}$ Morris, S. J., "Miniature Spy Planes: The Next Generation of Flying Robots," Frontiers of Engineering Reports on Leading-edge Engineering from the March 2002 NAE Symposium on Frontiers of Engineering, 2002, pp. 10-20.

${ }^{2}$ Sneyd, A., "Aerodynamic coefficients and longitudinal stability of sail aerofoils," J. Fluid Mech., Vol. 149, 1984, pp. 127146.

${ }^{3}$ Smith, R. and Shyy, W., "Computation of unsteady laminar flow over a flexible two-dimensional membrane wing," Physics of Fluids, Vol. 7, 1995, pp. 2175-2184.

${ }^{4}$ Smith, R. and Wright, J., "Simulation of the viscous aeroelastic response of a membrane airfoil to a harmonically varying freestream," 39th AIAA Aerospace Sciences Meeting bo Exhibit - AIAA 2001-0857, 2001, pp. 1-7.

${ }^{5}$ Dowell, E. H. and Hall, K. C., "Modeling of Fluid-Structure Interaction," Annual Review of Fluid Mech., Vol. 33, 2001, pp. $445-490$.

${ }^{6}$ Naudasher, E. and Rockwell, D., Flow-Induced Vibrations An Engineering Guide, A.A.Balkema, 1994.

${ }^{7}$ Dowell, E., Curtiss, H., Scanlan, R., and Sisto, F., A modern course in aeroelasticity, 2nd ed., Kluwer Academic Publisher, 1989.

${ }^{8}$ Lumley, J., "The Structure of Inhomogeneous Turbulent Flows," Atm. Turb. And Radio Wave Prop., Nauka, Moscow, Toulouse, France, edited by Yaglom and Tatarsky, 1967, pp. 166-178.

${ }^{9}$ Berkooz, G., Holmes, P., and Lumley, J., "The Proper Orthogonal Decomposition in the Analysis of Turbulent Flows," Annual Review of Fluid Mech., Vol. 25, 1993, pp. 539-575.

${ }^{10}$ Payne, F., Large Eddy Structure of a Turbulent Wake, Ph.D. thesis, Pennsylvania State University, 1966.
${ }^{11}$ Bakewell, P. and Lumley, J., "Viscous Sublayer and Adjacent Wall Region in Turbulent Pipe Flow," Physics of Fluids., Vol. 10, No. 3, 1978, pp. 1880-1889.

${ }^{12}$ Bonnett, J., Delville, J., Glauser, M., Antonia, R., Bisset, D., Cole, D., Fiedler, H., Garem, J., Hilberg, D., Jeong, J., Kevlahan, N., Ukeiley, L., and Vincendau, E., "Collaborative Testing of Eddy Structure Identification Methods in Free Turbulent Shear Flows," Experiments in Fluids, Vol. 25, 1998, pp. 197-225.

${ }^{13}$ Taylor, J. and Glauser, M., "Towards Practical Flow Sensing and Control via POD and LSE Based Low-Dimensional Tools," 2002 ASME Fluids Engineering Division Summer Meeting - FEDSM2002-31416, 2002, pp. 1-9.

${ }^{14}$ Hamstra, J. W. and Miller, D. N., "Active Flow Control Enabling Next-Generation Jet Propulsion Aerodynmaics," Frontiers of Engineering Reports on Leading-edge Engineering from the March 2002 NAE Symposium on Frontiers of Engineering, 2002, pp. 3-9.

${ }^{15}$ Laranko, Owners Manual for the Digital Strero PIV System, Laranko, 2002.

${ }^{16}$ Adrian, R., "On the Role of Conditional Averages in Turbulence Theory," Turbulence in Liquids: Proceedings of the Fourth Biennial Symposium on Turbulence in Liquids, J. Zakin and G. Patterson (Eds.) Science Press, Princeton, 1977, pp. 323-332.

${ }^{17}$ Cole, D., Glauser, M., and Guezennec, Y., "An Application of Stochastic Estimation to the Jet Mixing Layer," Phys. Fluids, Vol. 4, No. 1, 1991, pp. 192-194.

${ }^{18}$ Bonnet, J., Cole, D., Delville, J., Glauser, M., and Ukeiley, L., "Stochastic Estimation and Proper Orthogonal Decomposition: Complementary Techniques for Identifying Structure," Experiments in Fluids, Vol. 17, 1994, pp. 307-314.

${ }^{19}$ Schmit, R. and Glauser, M., Low Dimensional Tools for Flow-Structure Interaction Problems: Application to Micro Air Vehicles, Ph.D. thesis, Clarkson University, 2002.

${ }^{20}$ Hussain, A. K. M. F. and Hayakawa, M., "Eduction of large-scale organized structures in a turbulent plane wake," $J$. Fluid Mech., Vol. 180, 1987, pp. 193-229. 